\title{
On Using Gaussian Excitation Amplitudes to Improve the Antenna Array Radiation Characteristics
}

\author{
Mohamed F. A. Ahmed ${ }^{1,2}$, Osama M. Haraz ${ }^{1,3}$, Georges Kaddoum², Saleh A. Alshebili ${ }^{3}$,Abdel-Razik Sebak ${ }^{4}$ \\ Electrical Engineering Department ${ }^{1}$ \\ Faculty of Engineering, Assiut University, \\ Assiut, 71515 Egypt \\ osama.haraz@eng.edu.au.eg \\ LACIME Laboratory ${ }^{2}$ \\ ETS Engineering School, University of Québec, \\ Montreal, Quebec, Canada \\ mabdella@ualberta.ca, georges.kaddoum@etsmtl.ca \\ KACST Technology Innovation Center in RFTONICS ${ }^{3}$ \\ King Saud University, \\ Riyadh, Saudi Arabia \\ oharaz@ksu.edu.sa,dsaleh@ksu.edu.sa \\ Department of Electrical and Computer Engineering ${ }^{4}$ \\ Concordia University, \\ Montreal, Quebec H4G 2W1 Canada \\ abdo@ece.concordia.ca
}

\begin{abstract}
In this paper, new excitation amplitudes based on Gaussian distribution are used to improve the radiation characteristics of millimeter-wave patch array antenna operating at $30 \mathrm{GHz}$. To prove the concept, we compare different traditional excitation amplitudes with the Gaussian one for uniform linear array (ULA) and uniform planar array (UPA). We apply the Gaussian excitation amplitudes to 32 patch antenna elements for the ULA while we use $8 \times 8$ patch antenna elements for the UPA.
\end{abstract}

Keywords-Patch array; Gaussian distributions; Fifth generation (5G) wireless communications; Uniform linear array (ULA); Uniform planar array (UPA).

\section{INTRODUCTION}

Over the next few years, researchers are required to propose and implement technologies with higher data rates and much lower latencies than the ones available today to target the fifth generation $(5 \mathrm{G})$ mobile networks. However, with almost saturated bandwidth available for mobile communications, utilizing new spectrum becomes a necessity. To this end, millimeter-wave transmission is recently considered in addition to the microwave range currently used. The short wavelength in the millimeter-wave spectrum allows for smaller antennas and thus the ability to fabricate patch antenna arrays with large number of elements on a small chip. Consequently, more efficient beamforming and spatial user multiplexing become possible with this large number of antenna elements in the array. However, the performance critically depends on the radiation pattern of the antenna array.

Antenna arrays allows higher directivity with sidelobe level suppression and beamwidth reduction. An equally spaced antenna array has a periodic radiation pattern with deterministic side lobes. Moreover, grating lobes, which are sidelobes with levels equal to the main lobe, occur when the element spacing is much greater than a half wavelength. One solution to avoid grating is to use random arrays where the antenna elements are distributed over a linear, planar, or spherical aperture with unequal spacing $[1,2]$. The randomness of the array elements produce random sidelobes with levels lower than the main lobe level [3]. Based on the results of the theory of random arrays [13 ], the radiation pattern is equivalent to the characteristic function of the spatial distribution function of continues aperture excitation. Therefore, we have the ability to obtain any required radiation pattern by spatially distributing the array elements according to spatial distribution with the proper characteristic function. However, the exact radiation pattern is obtained when the number of the array elements reaches infinity. Furthermore, since the antenna element has finite physical dimension, there is a minimum distance allowed between any two elements. Consequently, random arrays have larger area than equally spaced arrays with the same number of elements.

In this paper, we propose an alternative solution to the random arrays and use uniformly spaced arrays with nonuniform excitation amplitudes. We propose Gaussian excitation amplitudes to feed patch antenna array. Thus, for a linear array, the excitation amplitudes are samples of the function

$$
f(d, \mu, \sigma)=\left(\frac{1}{\sigma \sqrt{2 \pi}}\right) e^{-\frac{(d-\mu)^{2}}{2 \sigma^{2}}}
$$

where $\mathrm{d}$ is the element coordinates, $\mu=0$, and $\sigma$ is selected based on the linear array length. The Gaussian distribution function has an exponentially decaying characteristic function and consequently results in similar decaying radiation pattern.

Both linear and planar arrays are considered in this paper where the planer case uses 2-dimensional Gaussian function. Simulation results have been obtained for radiation patterns, antenna gain, and sidelobe levels by using Computer Simulation Technology (CST) Microwave Studio simulation software program [4]. 


\section{32-ELEMENT UNIform LinEAR PATCh ANTENNA ARRAY WITH GAUSSIAN EXCITATION AMPLITUDES}

Let us first consider 32-element uniform linear patch antenna array with geometrical configuration as shown in Fig. 1. The array is uniform in spatial domain, so the patch antenna elements are equispaced. For the operating frequency of $f=30$ $\mathrm{GHz}$, the free-space wavelength will be $\lambda=10 \mathrm{~mm}$ only. Accordingly, the inter-element spacing is $d_{1}=d_{2}=\ldots=d_{N}=\lambda / 2$ $=5 \mathrm{~mm}$ and the total length of the 32-element uniform patch antenna array is $l=16 \lambda=160 \mathrm{~mm}$. The array is fed with nonuniform excitation amplitudes based on Gaussian distribution (1). It was shown in [5] that if the Gaussian function is truncated at $d=3 \sigma$ then the radiation pattern is almost equal to the Gaussian function with infinite support. This is because the function values for $d>3 \sigma$ are almost zero. Thus, the value of $\sigma$ is found from the equality $3 \sigma=l / 2$ and the proposed Gaussian amplitude weights are calculated from (1) as

$\left[\begin{array}{llllllll}0.0013 & 0.0024 & 0.0034 & 0.0052 & 0.0077 & 0.0117 & 0.0154 & 0.0222 \\ 0.0278 & 0.0358 & 0.0429 & 0.0511 & 0.0590 & 0.0659 & 0.0692 & 0.0732 \\ 0.0655 & 0.0604 & 0.0533 & 0.0454 & 0.0368 & 0.0295 & 0.0219 & 0.0721 \\ 0.0171 & 0.0124 & 0.0084 & 0.0050 & 0.0710 & 0.0036 & 0.0022 & 0.0012\end{array}\right]$

In order to verify the performance benefits of proposed Gaussian excitation amplitudes, the array is simulated using CST Microwave Studio [4]. The simulated E- and H-plane radiation patterns of the proposed 32-element patch antenna array with Gaussian excitation amplitudes are presented in Fig. 2 and Fig. 3. Curves for binomial, Chebychev, uniform cosine, cosine square and Taylor excitation amplitudes are also presented for comparison purposes. It can be seen from the figure that the Gaussian amplitude weights give good performance in terms of gain, sidelobe level, and front-to-back ratio. The detailed comparison among different amplitude weight distributions are summarized in Table I. The gain of the patch antenna array with Gaussian excitation amplitudes is about

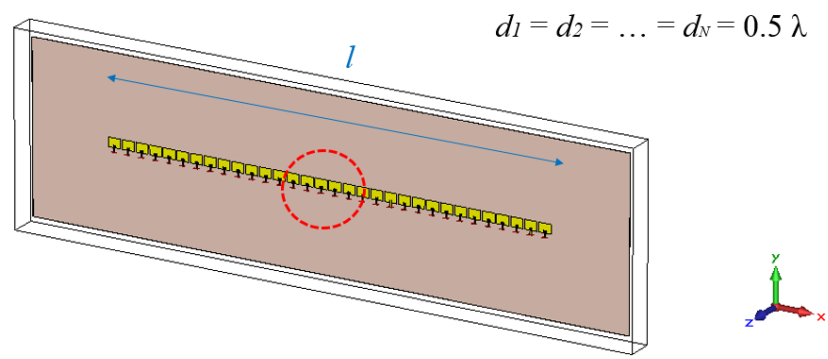

(a)

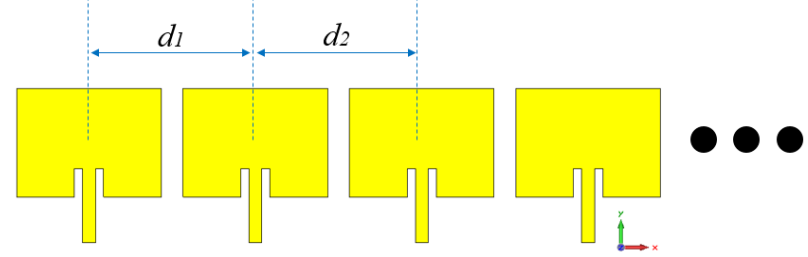

(b)

Fig. 1. Proposed 32-element linear patch antenna array (a) geometrical configuration, and (b) detailed view.
$17.9 \mathrm{dBi}$. The sidelobes are $-17.9 \mathrm{~dB}$ and $-18.8 \mathrm{~dB}$ for both $E$ plane and $H$-plane.

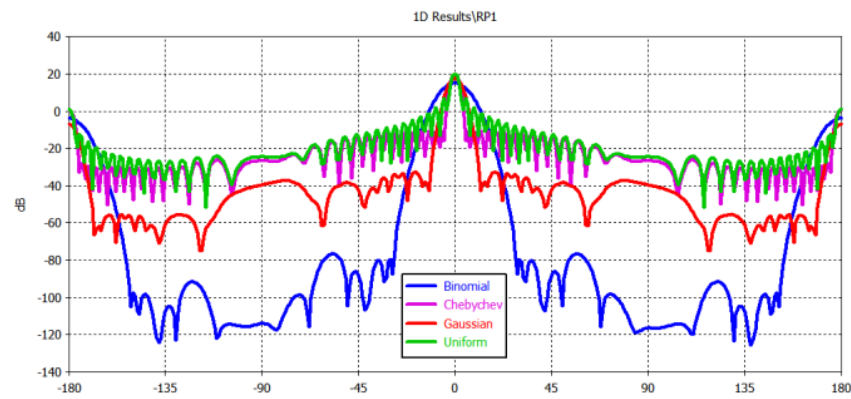

(a)

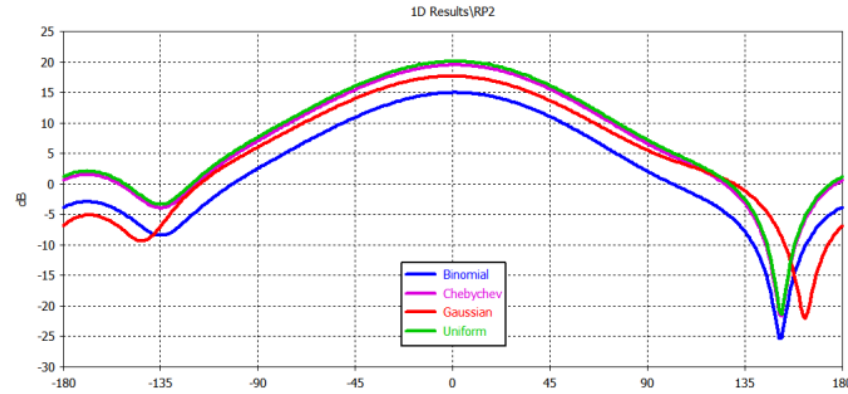

(b)

Fig. 2. Calculated (a) $H$-plane and (b) $E$-plane radiation patterns for the proposed 32-element uniform linear array with Gaussian amplitude weights. Curves for binomial, Chebychev and uniform amplitude weights are also presented for comparison purposes.

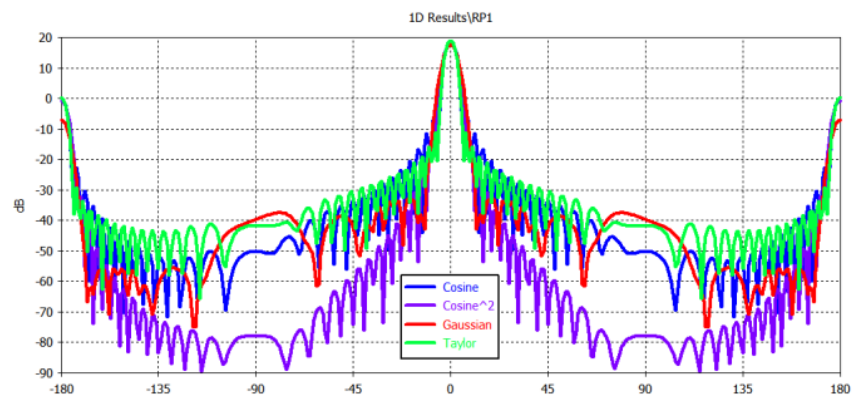

(a)

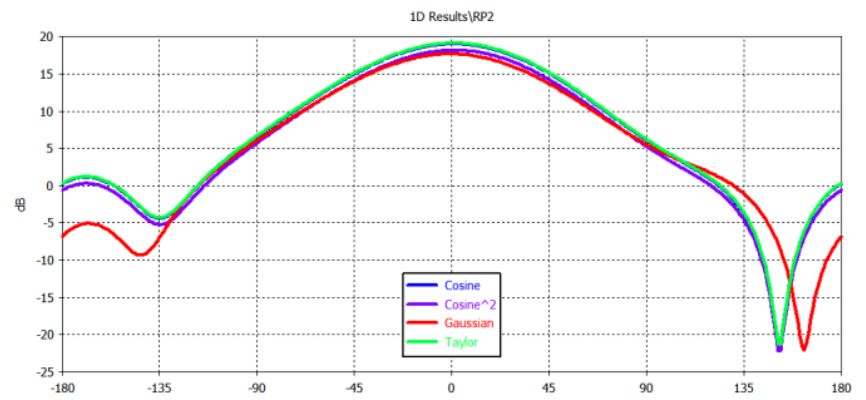

(b)

Fig. 3. Calculated (a) $H$-plane and (b) $E$-plane radiation patterns for the proposed 32-element uniform linear array with Gaussian amplitude weights. Curves for cosine, cosine ${ }^{\wedge} 2$ and Taylor amplitude weights are also presented for comparison purposes. 
TABLE I

PERFormance COMPARISONS AMONG DifFERENT AMPlitude WeIGHTS FOR THE 32-ELEMENT LINEAR PATCH ANTENNA ARRAY

\begin{tabular}{ccccc}
\hline \hline $\begin{array}{c}\text { Amplitude } \\
\text { weights }\end{array}$ & $\begin{array}{c}\text { Maximum } \\
\text { gain, dB }\end{array}$ & $\begin{array}{c}\text { Front-to- } \\
\text { back ratio, } \\
\mathrm{dB}\end{array}$ & $\begin{array}{c}\text { Sidelobe } \\
\text { level in } E \text { - } \\
\text { plane, dB }\end{array}$ & $\begin{array}{c}\text { Sidelobe } \\
\text { level in } H \text { - } \\
\text { plane, dB }\end{array}$ \\
\hline Binomial & 15.0 & 18.6 & -17.9 & -18.8 \\
Chebychev & 19.5 & 18.7 & -18.0 & -18.9 \\
Cosine & 19.0 & 18.6 & -17.9 & -18.8 \\
Cosine^2 & 18.2 & 18.6 & -17.9 & -18.8 \\
Taylor & 19.1 & 18.6 & -17.9 & -18.8 \\
Uniform & 20.1 & 18.7 & -18.0 & -13.3 \\
Gaussian & 17.9 & 18.6 & -17.9 & -18.8 \\
\hline \hline
\end{tabular}

\section{III. $8 \times 8$-ELEMENT UNIFORM PlanAR PATCH ANTENNA} ARRAY WITH GAUSSIAN EXCITATION AMPLITUDES

The geometrical configuration of the proposed $8 \times 8$ uniform planar patch antenna array is shown in Fig. 4. The planar array is uniform as well in spatial domain so the patch antenna elements are equispaced. The inter-element spacing along $\mathrm{x}$-axis is $d_{x}=\lambda / 2=5 \mathrm{~mm}$ and the inter-element spacing along $\mathrm{y}$-axis is $d_{y}=\lambda / 2=5 \mathrm{~mm}$. The length of the $8 \times 8$ uniform patch antenna array along the $\mathrm{x}$-axis and the $\mathrm{y}$-axis are the same and the physical dimension of the antenna array is $l_{x}=l_{y}=4 \lambda=40 \mathrm{~mm}$. The planar array is fed with non-uniform excitation amplitudes based on Gaussian distribution calculated, in a similar way as the linear array, from 2-dimensional Gaussian function as

$\left[\begin{array}{llllllll}0.0002 & 0.0007 & 0.0020 & 0.0034 & 0.0034 & 0.0020 & 0.0007 & 0.0020 \\ 0.0007 & 0.0034 & 0.0094 & 0.0156 & 0.0156 & 0.0094 & 0.0034 & 0.0007 \\ 0.0020 & 0.0094 & 0.0259 & 0.0432 & 0.0432 & 0.0259 & 0.0094 & 0.0020 \\ 0.0034 & 0.0156 & 0.0432 & 0.0720 & 0.0720 & 0.0432 & 0.0156 & 0.0034 \\ 0.0034 & 0.0156 & 0.0432 & 0.0720 & 0.0720 & 0.0432 & 0.0156 & 0.0034 \\ 0.0020 & 0.0094 & 0.0259 & 0.0432 & 0.0432 & 0.0259 & 0.0094 & 0.0020 \\ 0.0007 & 0.0034 & 0.0094 & 0.0156 & 0.0156 & 0.0094 & 0.0034 & 0.0007 \\ 0.0002 & 0.0007 & 0.0020 & 0.0034 & 0.0034 & 0.0020 & 0.0007 & 0.0002\end{array}\right]$

The array is simulated using CST and the simulated $E$ - and $H$-plane radiation patterns are presented in Fig. 5 and Fig. 6. Curves for binomial, Chebychev, uniform cosine, cosine square, and Taylor excitation amplitudes are also presented for comparison as well. It can be noticed that the Gaussian excitation amplitudes give good performance in terms of gain, side lobe level, and front-to-back ratio. The detailed comparison among different amplitude weight distributions are summarized in Table II.

TABLE II

PERFormance COMPARISONS AMONG DifFERENT AMPLITUdE WEIGHTS FOR THE 8X8 PLANAR PATCH ANTENNA ARRAY

\begin{tabular}{ccccc}
\hline \hline $\begin{array}{c}\text { Amplitude } \\
\text { weights }\end{array}$ & $\begin{array}{c}\text { Maximum } \\
\text { gain, dB }\end{array}$ & $\begin{array}{c}\text { Front-to- } \\
\text { back } \\
\text { ratio, dB }\end{array}$ & $\begin{array}{c}\text { Sidelobe } \\
\text { level in } E- \\
\text { plane, dB }\end{array}$ & $\begin{array}{c}\text { Sidelobe } \\
\text { level in } H- \\
\text { plane, dB }\end{array}$ \\
\hline Binomial & 17.32 & 34.50 & -34.5 & -34.5 \\
Chebychev & 20.37 & 35.86 & -30.6 & -32.0 \\
Cosine & 18.47 & 34.81 & -21.6 & -21.6 \\
Cosine^2 & 17.10 & 34.10 & -34.0 & -34.1 \\
Taylor & 20.00 & 35.64 & -31.6 & -32.6 \\
Uniform & 22.10 & 37.34 & -12.9 & -13.4 \\
Gaussian & 17.61 & 34.68 & -34.7 & -34.7 \\
\hline \hline
\end{tabular}

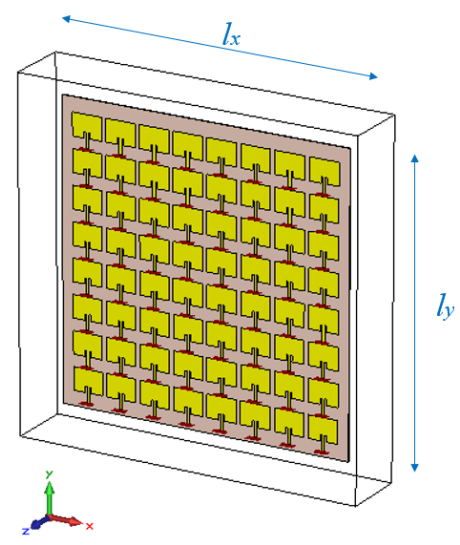

(a)

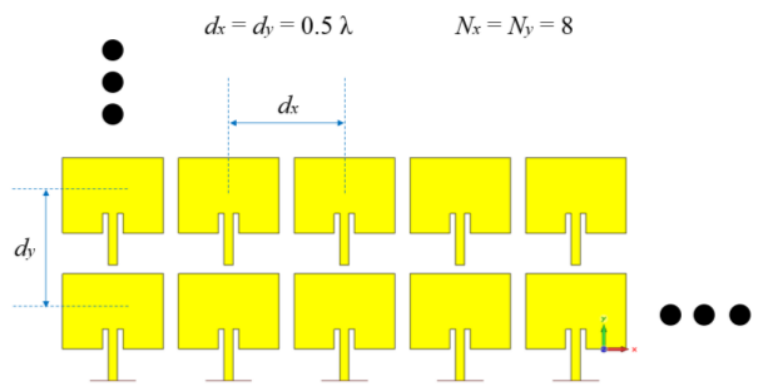

(b)

Fig. 4. Proposed $8 \times 8$ planar patch antenna array (a) geometrical configuration, and (b) detailed view.

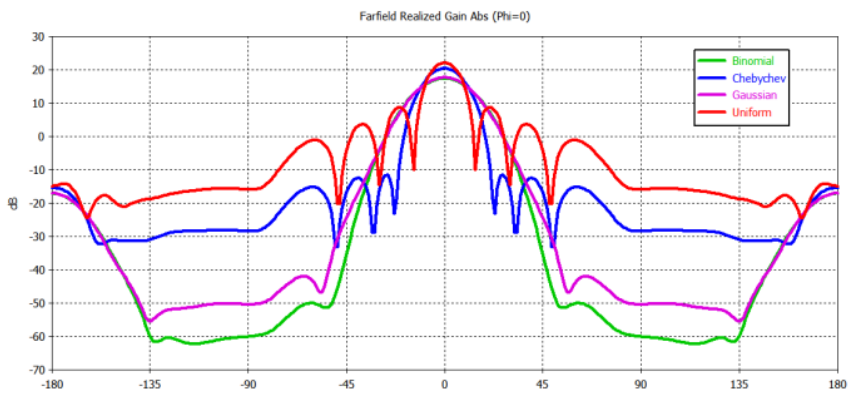

(a)

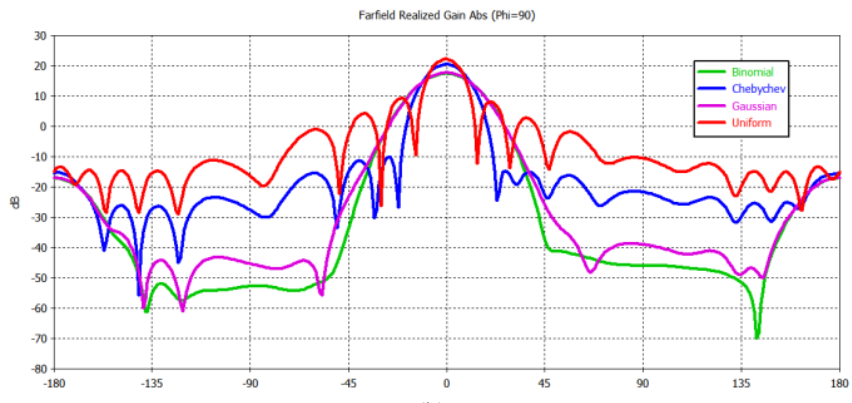

(b)

Fig. 5. Calculated (a) $H$-plane and (b) $E$-plane radiation patterns for the proposed 8x8 uniform planar array with Gaussian amplitude weights. Curves for binomial, Chebychev and uniform amplitude weights are also presented for comparison purposes. 


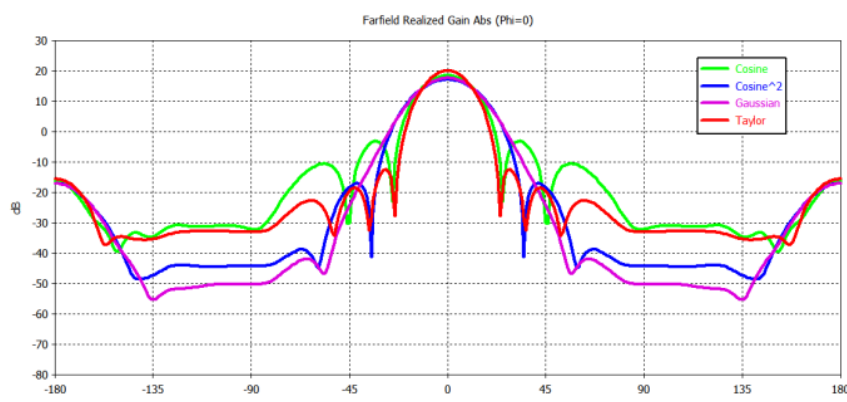

(a)

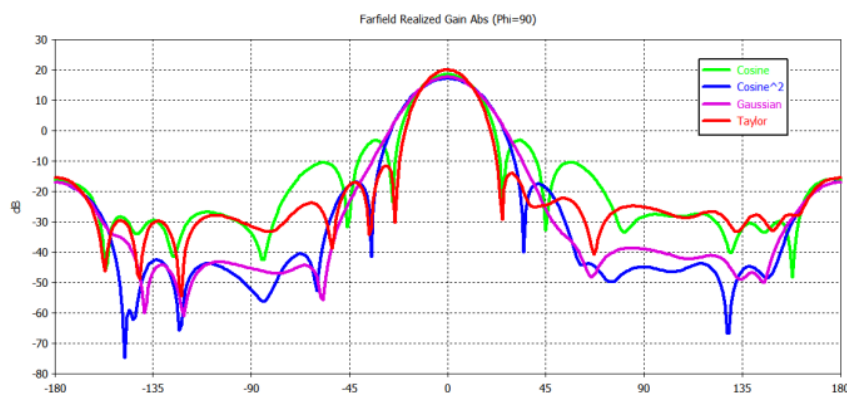

(b)

Fig. 6. Calculated (a) $H$-plane and (b) $E$-plane radiation patterns for the proposed $8 \times 8$ uniform planar array with Gaussian amplitude weights. Curves for cosine, $\operatorname{cosine} \mathrm{e}^{\wedge} 2$ and Taylor amplitude weights are also presented for comparison purposes.

\section{GAIN AND SIDELOBES LEVEL COMPROMISE FOR THE GAUSSIAN EXCITATION AMPLITUDES}

The excitation amplitudes are samples from the Gaussian distribution function where the equality $3 \sigma=l / 2$ is maintained to obtain the performance of a Gaussian function with infinite support. However, a truncated Gaussian function can be used instead which corresponds, for the same array dimensions, to a Gaussian distribution with larger $\sigma$. It is shown in [5] that larger $\sigma$ results in smaller beam width and thus higher directivity. The effect in our proposed array is having smaller beamwidth and higher sidelobes. Therfore, a compromise between the gain and sidelobes level can be obtained using the Gaussian excitation amplitudes. Due to the limited space, results for different $\sigma$ are not shown here.

\section{CONCLUSION}

Gaussian excitation amplitudes for patch antenna array operating in the millimeter-wave has been presented in this paper. The radiation characteristics at $30 \mathrm{GHz}$ were found for both 32-element uniform linear array and $8 \times 8$ uniform planar array. The proposed excitation amplitudes allows for better radiation control as compared to the uniform and traditional non-uniform excitation amplitudes.

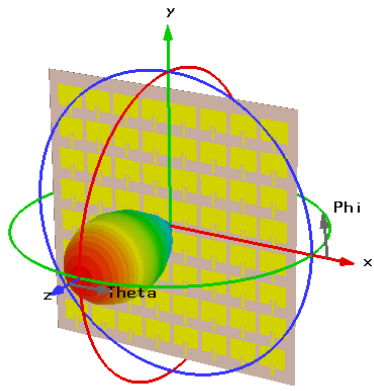

(a)

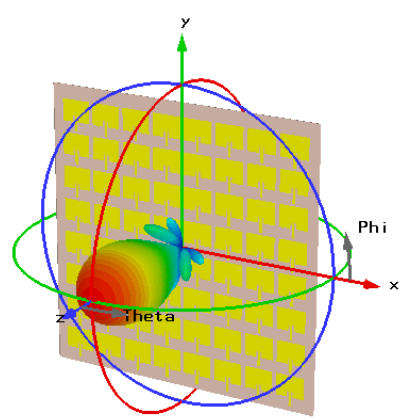

(c)

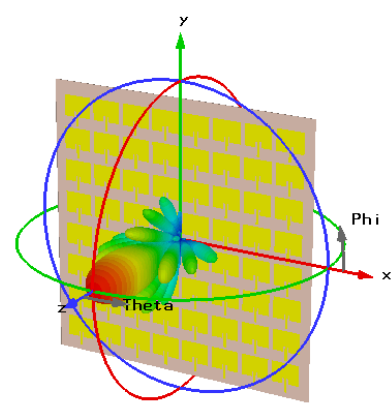

(e)

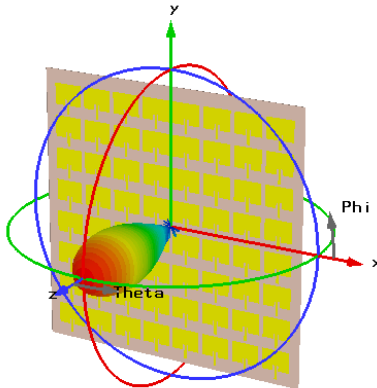

(b)

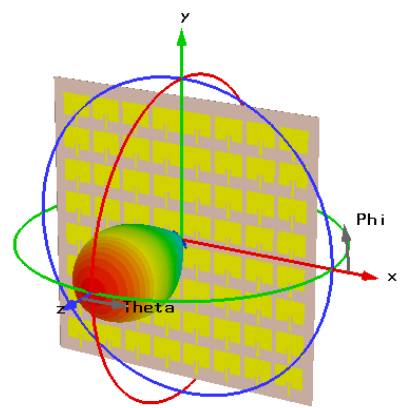

(d)

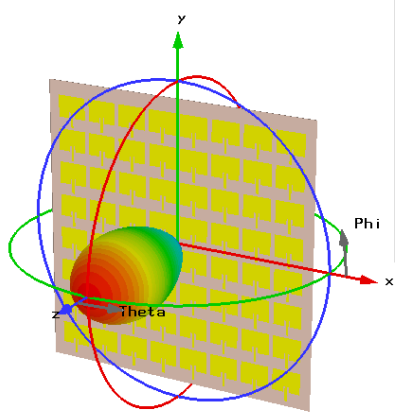

(f)
Fig. 7. Calculated 3D radiation patterns for the proposed $8 \times 8$ uniform planar array with (a) binomial, (b) Chebychev, (c) cosine, (d) $\operatorname{cosine}^{\wedge} 2$, (e) uniform, and (f) Gaussian amplitude weights.

\section{REFERENCES}

[1] Y. Lo, "A mathematical theory of antenna arrays with randomly spaced elements," IEEE Transactions on Antennas and Propagation, vol. 12, no. 3, pp. 257-268, May 1964.

[2] A. Panicali and Y. Lo, "A probabilistic approach to large circular and spherical arrays," IEEE Transactions on Antennas and Propagation, vol. 17 , no. 4, pp. $514-522$, Jul. 1969.

[3] M. Donvito and S. Kassam, "Characterization of the random array peak sidelobe," IEEE Transactions on Antennas and Propagation, vol. 27, no. 3, pp. 379 - 385, May 1979.

[4] CST Microwave Studio, version2012B.02, CST Microwave Studio, Wellesley Hills, MA, 2012.

[5] M. F. A. Ahmed and S. A. Vorobyov "Collaborative beamforming for wireless sensor networks with Gaussian distributed sensor nodes," IEEE Transactions on Wireless Communications, vol. 8, no. 2, pp. 638-643, 2009 . 